\title{
Beta-carotene prevents the spermatogenic disorders induced by exogenous scrotal hyperthermia through modulations of oxidative stress, apoptosis, and androgen biosynthesis in mice
}

\author{
Jung-Min Yon ${ }^{1}$, Jae Seung Kim ${ }^{1}$, Chunmei Lin², Seul Gi Park¹, Lee Wha Gwon ${ }^{1}$, Jong-Geol Lee', \\ In-Jeoung Baek ${ }^{3}$, Sang-Seop Nahm ${ }^{4}$, Sang-Yoon Nam ${ }^{1, *}$ \\ ${ }^{I}$ College of Veterinary Medicine, Chungbuk National University, Cheongju 28644, Korea \\ ${ }^{2}$ College of Chinese Medicinal Materials, Jilin Agricultural University, Changchun, Jilin 130-118, China \\ ${ }^{3}$ Asan Institute for Life Sciences, University of Ulsan College of Medicine, Seoul 05505, Korea \\ ${ }^{4}$ College of Veterinary Medicine, Konkuk University, Seoul 050029, Korea
}

\begin{abstract}
We investigated whether $\beta$-carotene ( $\beta-\mathrm{CA})$ or ellagic acid (EA), originating from various fruits and vegetables, has a preventive effect against male infertility induced by exogenous scrotal hyperthermia. ICR adult mice were intraperitoneally treated with $10 \mathrm{mg} / \mathrm{kg}$ of $\beta$-CA or EA daily for 13 days consecutively. During this time, mice were subjected to transient scrotal heat stress in a water bath at $43^{\circ} \mathrm{C}$ for $20 \mathrm{~min}$ on day 7, and their testes and blood were obtained on day 14 for histopathologic and biochemical analyses. Heat stress induced significant testicular weight reduction, germ cell loss and degeneration, as well as abnormal localization of phospholipid hydroperoxide glutathione peroxidase (PHGPx) and manganese superoxide dismutase (MnSOD) in spermatogenic and Leydig cells. Heat stress also altered the levels of oxidative stress (lipid peroxidation, SOD activity, and PHGPx, MnSOD, and HIF-1 $\alpha$ mRNAs), apoptosis (Bax, Bcl-xL, caspase 3, NF- $\kappa B$, and TGF- $\beta 1$ mRNAs), and androgen biosynthesis (serological testosterone concentration and $3 \beta$-hydroxysteroid dehydrogenase mRNA) in testes. These changes were all improved significantly by $\beta$-CA treatment, but only slightly improved by EA treatment. These findings indicate that $\beta$-CA, through modulations of oxidative stress, apoptosis, and androgen biosynthesis, is a potent preventive agent against testicular injuries induced by scrotal hyperthermia.
\end{abstract}

Keywords: $\beta$-carotene, ellagic acid, spermatogenic disorders, scrotal hyperthermia, testes

Jung-Min Yon and Jae Seung Kim contributed equally to this work.

*Corresponding author

Sang-Yoon Nam

Laboratory of Veterinary Anatomy, College of Veterinary Medicine, Chungbuk National University, Cheongju 28644, Korea

E-mail: synam@cbu.ac.kr

Tel: $+82-43-261-2596$

Fax: +82-43-271-3246

ORCID

Jung-Min Yon

https://orcid.org/0000-0002-1482-5586

Jae Seung Kim

https://orcid.org/0000-0003-2912-2610

Chunmei Lin

https://orcid.org/0000-0003-0738-2120

Seul Gi Park

https://orcid.org/0000-0002-8731-1113

Lee Wha Gwon

https://orcid.org/0000-0001-7373-0678

Jong-Geol Lee

https://orcid.org/0000-0002-4312-6282

In-Jeoung Baek

https://orcid.org/0000-0002-0641-7208

Sang-Seop Nahm

https://orcid.org/0000-0002-0519-580X

Sang-Yoon Nam

https://orcid.org/0000-0001-7576-6543

Conflict of Interest

The authors declare no conflicts of interest.

Received: February 21, 2019

Revised: May 28, 2019

Accepted: May 30, 2019

\section{Introduction}

Mammalian testes are located in an external scrotum; therefore, most mammal testes are $2-8^{\circ} \mathrm{C}$ below normal body temperature due to their presence outside the body. This lower temperature provides optimal spermatogenesis results [1]. However, modern lifestyles and environmental toxins such as cigarette smoking and cadmium exposure can induce scrotal hyperthermia and abnormal testicular thermoregulation (varicocele), resulting in male infertility. Indeed, testicular heating caused by modern lifestyle factors such as going to a sauna or spa, wearing tight underwear, using a laptop on one's lap, driving, and using heating pads could be harmful to spermatogenesis [2-5]. In patients with varicocele-related infertility, the high temperature of the testis causes gonad cell DNA damage, apoptotic cell death, and abnormal hormone balance [6]. About $20 \%$ of modern couples worldwide are infertile, and approximately half of these cases are male related [7]. Accordingly, there is a need to identify effective substances that can prevent male infertility.

A recent study showed that experimental scrotal heat stress (HS) in mice causes a hypoxic state and severe oxidative damage leading to a remarkable reduction in blood circulation in the testis, which leads to spermatogenesis disorders [8]. Hypoxic conditions of testes have been shown to result in apoptosis and adverse effects on Leydig cell (LC) competence and therefore spermatogenesis [9]. A large amount of free radicals and reactive oxygen species (ROS) produced in response to endogenous or exogenous toxic agents such as superoxide anion and hydrogen peroxide can be harmful to spermatogenesis and result in hindered male fertility in response to DNA injury [10,11]. Fur- 
thermore, LCs that produce steroids in the presence of luteinizing hormone (LH) are very sensitive to HS [12]. The reduction of testosterone production by selective destruction of LCs induces the death of spermatogenic cells via apoptosis in the rat testis [11], and follicle stimulating hormone, LH, and testosterone are known to be essential survival factors for testicular cells [13]. Therefore, bioactive agents originating from natural materials that have antioxidative, anti-apoptotic, and androgen biosynthetic activities might be useful in the prevention of testicular disorders induced by HS.

Carotenoids are natural pigments ranging in color from pale yellow to red that are produced by plants and fruits and known to contribute to the prevention of a variety of diseases. Three carotenoids, beta-carotene ( $\beta$-CA), alpha-carotene, and beta-cryptoxanthin, have vitamin $\mathrm{A}$ activity, and $\beta$ $\mathrm{CA}$ is one of the primary carotenoids consumed by humans and the most widely studied $[14,15]$. The biological level of $\beta$-CA is associated with biomarkers implicated under cardiometabolic conditions and cancer [16]. $\beta$-CA not only exerts anti-inflammatory activity by blocking redox-based nuclear factor kappa-light-chain-enhancer of activated B cells (NF$\kappa \mathrm{B})$ activation but also shows an antioxidative effect that increases cellular antioxidant enzyme activity and reduces oxidative stress $[17,18]$.

Ellagic acid (EA), which exists in a hydrolyzable tannin form known as ellagitannin in plants, is a natural polyphenol present in grapes, cranberries, raspberries, strawberries, blueberries, pomegranates, vegetables, and nuts. EA exerts chemopreventive effects via its ROS-reducing, anti-apoptotic, and anti-inflammatory properties [19].

In the current study, we investigated the effects of $\beta-\mathrm{CA}$ and EA on spermatogenic disorders induced by experimentally applied transient scrotal HS in the testes of adult mice. The regulatory mechanisms associated with the effects of $\beta$ $\mathrm{CA}$ and EA on the spermatogenic potential reduced by HS were also studied.

\section{Materials and Methods}

\section{Animals and chemicals}

Male ICR mice ( 8 weeks old; Koatech, Korea) were raised in a well-ventilated room kept at a temperature of $21^{\circ} \mathrm{C} \pm 2^{\circ} \mathrm{C}$ and a relative humidity of $55 \% \pm 10 \%$ on a $12 \mathrm{~h}$ light/dark cycle. The mice used in the experiment were provided with feed and water ad libitum. Animal experiments were performed according to the Guide for the Care and Use of Animals of the Chungbuk National University Animal Care Committee (CBNUA-469-13-02).

EA and $\beta$-CA purchased from Sigma (USA) were dissolved in isotonic saline and corn oil, respectively.

\section{Experimental design}

Mice were separated into four experimental groups: normal control (con), HS alone (HS), $10 \mathrm{mg} / \mathrm{kg}$ EA treatment with $\mathrm{HS}$ (HS+EA), and $10 \mathrm{mg} / \mathrm{kg} \beta-\mathrm{CA}$ treatment with $\mathrm{HS}$
(HS $+\beta-C A)$. Each group included 20 animals and the best effective quantity and route of EA or $\beta$-CA treatment was determined by our preliminary studies. EA and $\beta$-CA treatments were intraperitoneally administered once a day for 13 consecutive days. Additionally, HS application to the mice was conducted once on day 7 under intraperitoneal anesthesia with Zoletil (Virbac Korea Co. Ltd., Korea). Briefly, each mouse was sedated, after which they were immersed to below the forelimbs in a $43^{\circ} \mathrm{C}$ water bath for $20 \mathrm{~min}$. Control mice treated with each vehicle were anesthetized with Zoletil and then immersed in a water bath at room temperature. Following HS application, mice were dried, checked for scrotal damage, and then returned to their cages.

There was no damage to the scrotal skin in mice after HS treatment. At day 7 after HS, mice were autopsied after being sacrificed by inhalation anesthesia with diethyl ether. Testes and blood were then obtained for biochemical or histopathological investigations.

\section{Body and testicular weights}

Body and testicular weights of each animal were measured from day 1 to the end of the experiment (prior to sacrifice).

\section{Tissue processing and immunohistochemistry}

The testes were fixed with Bouin's fixative, followed by alcohol dehydration and paraffin embedding. After paraffin embedment, $4 \mu \mathrm{m}$ sections were stained with hematoxylin and eosin for histopathologic evaluation. For immunohistochemical analysis, the pretreatment process to reduce nonspecific reactions in the tissues, the reaction process for specific antibodies, the color development process, and the dehydration process using alcohol and xylene were performed as described in our previous paper [20]. In this study, a phospholipid hydroperoxide glutathione peroxidase (PHGPx; Abcam [ab102530; 1:50]; UK) antibody, a VECTASTAIN Elite ABC kit (PK-6101; rabbit IgG; Vectastain Elite ABC kit; Vector, USA), and diaminobenzidine (Vector) were used for the detection of specific antioxidant enzymes expressed in spermatogenic cells.

\section{Quantitative PCR}

Quantitative real-time PCR was conducted according to the method described in our previous paper [21]. Briefly, total RNA was extracted from a mouse testis to synthesize cDNA, after which quantitative real-time PCR was performed. Each PCR was amplified using the following primers: PHGPx, manganese superoxide dismutase (MnSOD), BCL2-associated $\mathrm{X}$ protein (Bax), B-cell lymphoma-extra large (Bcl-xL), caspase $3, \mathrm{NF}-\kappa \mathrm{B}$, transforming growth factor-beta 1 (TGF$\beta 1$ ), hypoxia inducible factor- $\alpha$ (HIF- $1 \alpha)$, 3 $\beta$-hydroxysteroid dehydrogenase (3$\beta$-HSD), and $\beta$-actin (Table 1). Triplicate data were examined by performing six independent analyses according to a comparative $\mathrm{Ct}$ method [22].

\section{Lipid peroxidation determination}

Lipid peroxidation was assessed in mouse testes according 
Table 1. Primers used for quantitative real-time polymerase chain reaction analysis

\begin{tabular}{llll}
\hline \hline \multirow{2}{*}{ Gene } & \multirow{2}{*}{ Accession no. } & \multicolumn{1}{c}{ Forward } & Primer $\left(5^{\prime}-3^{\prime}\right)$ \\
\cline { 3 - 4 } & & \multicolumn{1}{c}{ Reverse } \\
P-actin & NM_008084 & TTTCCAGCCTTCCTTCTTGGGTATG & CACTGTGTTGGCATAGAGGTCTTTAC \\
MnSOD & NM_008162 & TAAGAACGGCTGCGTGGT & GTAGGGGCACACACTTGTAGG \\
Bax & NM_013671 & GGAGCAAGGTCGCTTACAGA & GTGCTCCCACACGTCAATC \\
Bcl-xL & NM_009743 & TGACCACCTAGAGCCTTGGA & CACGGAGGAAGTCCAGTGTC \\
caspase3 & NM_009810 & AAAGCCGAAACTCTTCATCAT & TGTTCCCGTAGAGATCCACAA \\
NF- KB & NM_008689 & CACTGCTCAGGTCCACTGTC & GTCCCACTGTCTGTCTCA \\
TGF $\beta$ & NM_011577 & TGGAGCAACATGTGGAACTC & CTGTCACTATCCCGGAGTTCA \\
HIF-1 $\alpha$ & NM_010431 & CACCAGACAGAGCAGGAA & CAGCAGCCGGTTACCAAG \\
$3 \beta-H S D$ & NM_153193 & GACTGCTGACACACCACAC & TCAGGAACAGTATTTCTTTGATTCA \\
\hline
\end{tabular}

to our previous method [21]. Briefly, the concentration of thiobarbituric acid-reactive species was measured in the testis and expressed as malondialdehyde (MDA) equivalents. Data were obtained from five independent experiments.

\section{SOD activity assay}

Total SOD activity in the testes was measured using a SOD assay kit (Dojindo Laboratories, Japan) according to our previous method [23]. All experimental data were obtained five times independently.

\section{Testosterone level analysis}

Mouse blood collected from the abdominal aorta was centrifuged at $1000 \times g$ for 14 minutes to acquire supernatant. The supernatant was preserved at $-70^{\circ} \mathrm{C}$ until use. The testosterone concentration was measured in the obtained serum using an ELISA kit for mouse testosterone (Cusabio, China).

\section{Statistical analysis}

Differences among groups were assessed by one-way ANOVA and Tukey's multiple comparison test. A $p<0.05$ was considered statistically significant. Overall data were expressed as mean \pm standard error (SEM) values. All statistical assays were performed using SPSS for Windows, version 10.0 (SPSS Inc., USA).

\section{Results}

\section{Relative testicular weights}

Body weights showed no marked changes among groups during the experimental periods (data not shown). Measurement of relative testicular weight to body weight on day 7 after HS revealed a significant decrease in testis weight (Fig. 1). Specifically, the testis weight of the HS groups was significantly lower $(0.42 \pm 0.04$-fold, $p<0.05)$ than that of the control group; however, HS combined with $\beta$-CA administration led to a significant increase in weight by $0.79 \pm 0.05$-fold $(p<0.05)$ relative to that of the HS only group.

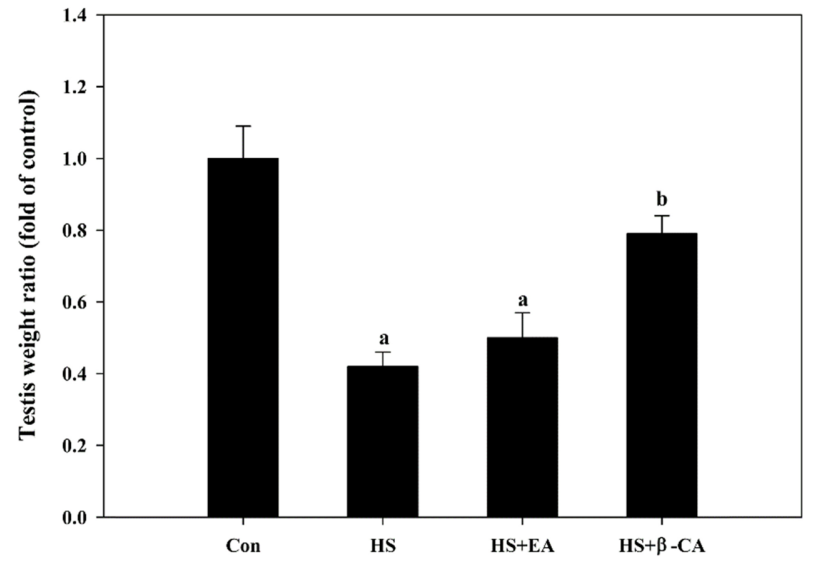

Fig. 1. Effects of $\beta$-CA or EA on HS-induced testicular weight changes. EA and $\beta$-CA were administered intraperitoneally once a day for 2 weeks and relative testicular weight was measured on day 7 after HS. Data are presented as mean \pm standard error values $(\mathrm{n}=20)$. a; vs. normal control (Con) group. $\mathrm{b} ;$ vs. HS group at $p<0.05$.

Con, normal control; HS, heat stress; EA, ellagic acid; $\beta$-CA, $\beta$ carotene.

\section{Histopathological results}

Histopathological examination revealed that the testes of the control group had normal features and spermatogenesis, containing abundantly normal spermatogenic cells in the seminiferous tubules (Fig. 2A). Conversely, the HS group showed diffuse and severe atrophy of seminiferous tubules characterized by loss of spermatogenic cells, cellular degeneration, multinucleated cell formation, and vacuolation; moreover, the tubules were smaller in diameter than those of the normal tubules of the control group. Furthermore, the basement membrane of seminiferous tubules was thickened and LCs in interstitial tissues were also degenerative (Fig. 2B). However, this damage was less severe in the HS+EA group (Fig. 2C) than the HS group. Furthermore, the HS+ $\beta$ CA group showed a dramatic improvement in histological appearance relative to that of the HS group (Fig. 2D). The relative severities of histopathological findings are shown in 
Table 2. The severities of histopathological findings

\begin{tabular}{|c|c|c|c|c|}
\hline \multirow{2}{*}{ Histopathological findings } & \multicolumn{4}{|c|}{ Group $^{*}$} \\
\hline & Con & HS & HS+EA & $\mathrm{HS}+\beta-\mathrm{CA}$ \\
\hline Loss of spermatogenic cells & - & +++ & ++ & $+/-$ \\
\hline Degeneration of spermatogenic cells & - & ++ & + & + \\
\hline Multinucleated giant cell formation & - & +++ & ++ & $+/-$ \\
\hline Vacuolations of seminiferous tubules & - & +++ & + & $+/-$ \\
\hline Thickened basal membranes of seminiferous tubules & - & +++ & + & $+/-$ \\
\hline Degeneration of Leydig cells in interstitial tissues & - & $+1+$ & ++ & + \\
\hline
\end{tabular}

Con, normal control; HS, heat stress; EA, ellagic acid; $\beta$-CA, $\beta$-carotene.

*Severities: severe $(+++)$, moderate $(++)$, mild $(+)$, normal $(-)$.

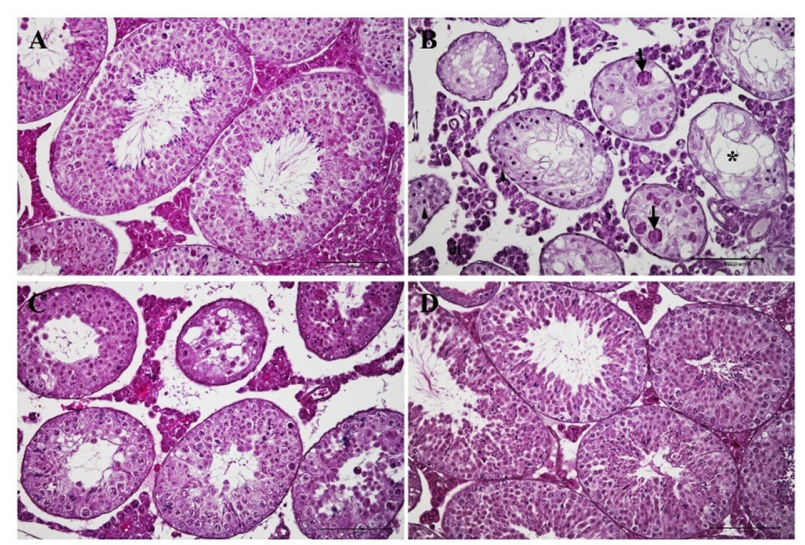

Fig. 2. Preventive effects of $\beta$-CA or EA against spermatogenic disorders induced by exogenous scrotal HS. Hematoxylin and eosin-stained testes from the normal control group (A), HS group (B), HS plus EA group (C) and HS plus $\beta$-CA group (D). The testes exposed to HS showed multinucleated giant cells (arrows) and atrophic spermatogenic cells (arrowheads), small to large vacuoles (asterisks) and irregular spaces in seminiferous tubules, thickened basal membranes, and degenerative Leydig cells in interstitial tissues. However, when mice were preand post-treated with $\beta$-CA under HS, testes revealed almost normal features (D), whereas severe spermatogenic disorders were detected in the HS plus EA group (C). Bar: $100 \mu \mathrm{m}$. $\beta$ CA, $\beta$-carotene; EA, ellagic acid; HS, heat stress.

Table 2. Pre- and post-treatment of $\beta$-CA in conjunction with HS restored the testes to a structure similar to that of the normal group, resulting in preservation of the tubular structure and the presence of many tubules including plentiful spermatids and spermatocytes.

\section{Abnormal distribution of testicular PHGPx induced by $\mathrm{HS}$ is recovered by $\beta-\mathrm{CA}$ co-treatment}

As it is a biomarker for spermatogenic cells, PHGPx localization was examined in testes by undertaking immunohistochemical analysis (Fig. 3). PHGPx in the normal testes was strongly detected in round and elongated spermatids and in spermatozoa, but was slightly observed in interstitial LCs (Fig. 3A). However, PHGPx levels in testes exposed to HS was greater in degenerative germ cells of seminiferous tubules

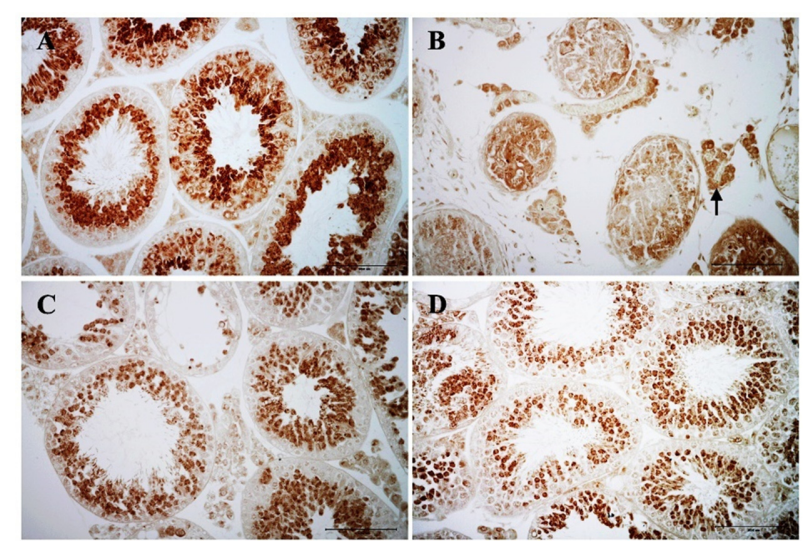

Fig. 3. Testicular localization of PHGPx following by exogenous scrotal HS and/or $\beta$-CA or EA co-treatment. PHGPx immunoreactivity in the testes from the normal control group (A), HS group (B), HS plus EA group (C) and HS plus $\beta$-CA group (D). PHGPx was detected at high levels in round and elongated spermatids in seminiferous tubules and at low levels in interstitial Leydig cells (A, C, and D), but strongly observed in interstitial Leydig cells of HS group (arrow; B). Bar: $100 \mu \mathrm{m}$. PHGPx, phospholipid hydroperoxide glutathione peroxidase; HS, heat stress; $\beta$-CA, $\beta$-carotene; EA, ellagic acid.

and interstitial LCs (Fig. 3B). Conversely, these abnormal distributions of PHGPx in the testes in response to HS treatment were restored to almost normal by the administration of $\beta$-CA (Fig. 3D); however, strong PHGPx immunoreactivity remained in the multinucleated giant cells in the atrophic seminiferous tubules of the HS+EA group (Fig. 3C).

\section{Expression levels of PHGPx and MnSOD mRNAs}

The mRNA levels of PHGPx and MnSOD were significantly lower in the HS group (0.52- and 0.72-fold, respectively) than in the control group (1-fold, $p<0.05)$. However, compared to the control group (1.0-fold), the decrease in PHGPX mRNA level induced by HS increased significantly in response to treatment with EA or $\beta$-CA, 0.84- and 1.42fold increases, respectively (Fig. 4A). Furthermore, treatment with EA or $\beta-\mathrm{CA}$ recovered the MnSOD mRNA level reduced by HS to approximately 1.00 - and 1.16-fold, respectively, compared to that of the control group (1.0-fold, Fig. 4B). 

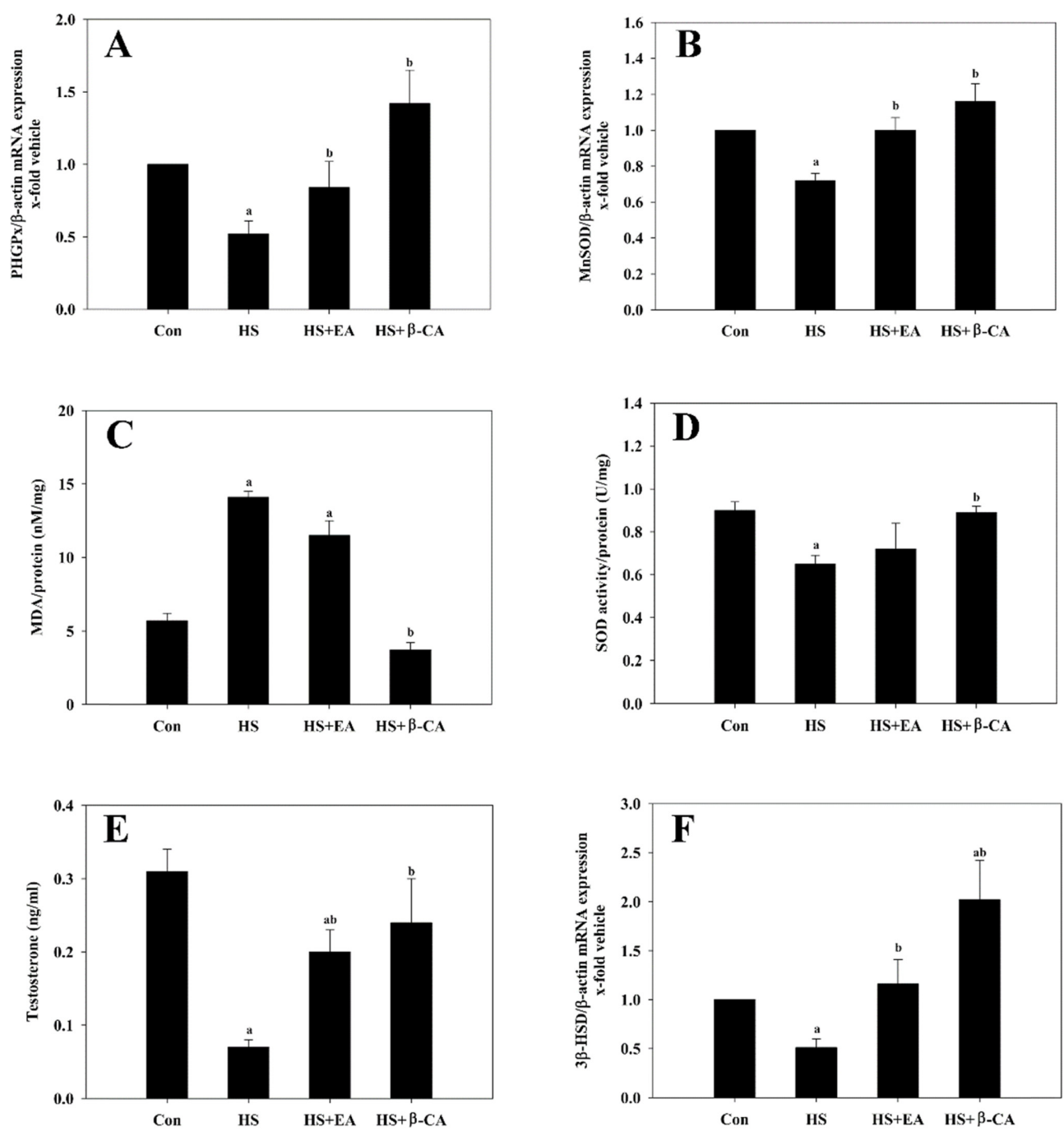

Fig. 4. Oxidative stress (A-D) and androgen biosynthesis (E, F) patterns in testes following exogenous scrotal HS and/or $\beta$-CA or EA co-treatment. The expression of PHGPx and MnSOD mRNA and SOD activity was significantly restored by co-treatment with $\beta$-CA or EA relative to that in the HS group. Co-treatment with $\beta$-CA leads to a significant decrease in MDA levels compared to that in the HS group. Testosterone and 3 $\beta$-HSD mRNA levels are significantly restored by EA or $\beta$-CA co-treatment. Data are presented as mean \pm standard error values $(\mathrm{n}=10)$. $\mathrm{a}$; $v s$. con group. $\mathrm{b} ; v s$. HS group at $p<0.05$.

Con, normal control; HS, heat stress; EA, ellagic acid; $\beta$-CA, $\beta$-carotene; PHGPx, phospholipid hydroperoxide glutathione peroxidase.

\section{Lipid peroxidation levels}

The level of testicular lipid peroxidation was assessed by assessing MDA equivalents. As shown in Fig. 4C, the MDA level $(14.1 \pm 0.4 \mathrm{nmol} / \mathrm{mg})$ of the HS group was significantly higher than that of the control group $(5.7 \pm 0.5 \mathrm{nmol} /$ $\mathrm{mg} ; p<0.05)$. In contrast, co-treatment with $\beta$-CA significantly decreased the MDA level to $3.7 \pm 0.5 \mathrm{nmol} / \mathrm{mg}$ relative to that of the HS group $(p<0.05)$, and similar to that of the control group, whereas the MDA level in the HS+EA group showed only a slight decrease $(11.5 \pm 1.0 \mathrm{nmol} / \mathrm{mg})$ from that of the HS group.

\section{SOD activity levels}

Testes exposed to HS showed significantly reduced SOD activity $(0.65 \pm 0.04 \mathrm{U} / \mathrm{mg}$ protein $)$ relative to that of the con- trol group $(0.90 \pm 0.04 \mathrm{U} / \mathrm{mg}$ protein; $p<0.05)$. However, co-treatment with $\beta$-CA significantly elevated the SOD activity level $(0.89 \pm 0.03 \mathrm{U} / \mathrm{mg}$ protein; $p<0.05)$ relative to that of the HS group, whereas the SOD activity level in the HS+ EA group showed only a slight increase $(0.72 \pm 0.12 \mathrm{U} / \mathrm{mg}$ protein; Fig. 4D) from that of the HS group.

\section{Testosterone concentration and expression patterns

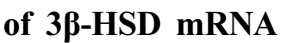

The serological testosterone level in the HS group was reduced significantly $(0.07 \pm 0.01 \mathrm{ng} / \mathrm{mL})$ compared to that of the control group $(0.31 \pm 0.03 \mathrm{ng} / \mathrm{mL})$. However, treatment with EA or $\beta$-CA in conjunction with HS led to significant recovery to $0.20 \pm 0.06$ and $0.24 \pm 0.1 \mathrm{ng} / \mathrm{mL}$, respectively $(p<0.05$; Fig. 4E). 

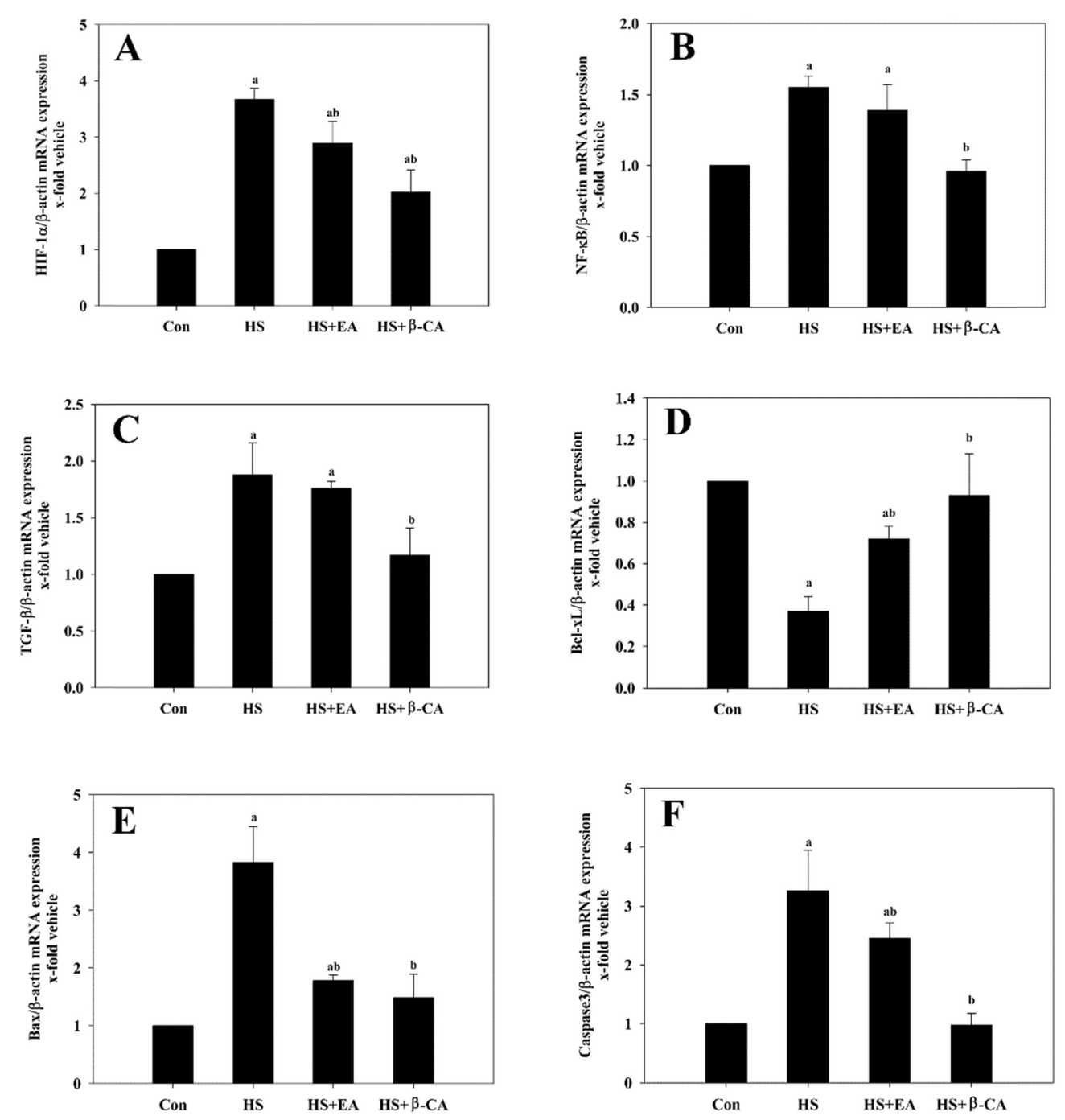

Fig. 5. The mRNA expression of hypoxia and apoptosis-modulating proteins: HIF-1 $\alpha$ (A), NF- $\kappa B$ (B), TGF- $\beta 1$ (C), Bcl-xL (D), Bax (E), and caspase 3 (F) following exogenous scrotal HS and/or $\beta$-CA) or EA co-treatment. The Bcl-xL mRNA level is significantly recovered by co-treatment with $\beta$-CA or EA compared to that in the HS group. Moreover, mRNA levels of HIF-1 $\alpha$, NF- $\kappa$, TGF$\beta 1$, Bax, and caspase 3 are significantly upregulated after HS exposure, but co-treatment with EA or $\beta$-CA significantly reduced the HS-elevated mRNA expression of hypoxia and apoptosis-related proteins. Data are presented as mean \pm standard error values $(\mathrm{n}=10)$. a; vs. Con group. b; vs. HS group at $p<0.05$.

Con, normal control; HS, heat stress; EA, ellagic acid; $\beta$-CA, $\beta$-carotene.

To evaluate whether LCs function normally following HS treatment, 3 $\beta$-HSD mRNA levels were investigated in the testes after HS treatment and/or co-treatment with EA or $\beta$ CA by using real-time PCR. The $3 \beta$-HSD mRNA following HS was significantly decreased when compared to that of the control group (0.51-fold of the control group; $p<0.05)$, but co-treatment with EA or $\beta$-CA significantly increased the $3 \beta$ HSD mRNA level to 1.16- and 2.02-fold, respectively ( $p<$ 0.05; Fig. 4F).

Gene expression patterns of hypoxia- and apoptosismodulating proteins

The HIF-1 $\alpha$ mRNA levels significantly increased in the
HS group (3.67-fold) when compared to that of the control group. However, treatment with EA or $\beta$-CA led to significant attenuation of HIF- $1 \alpha$ mRNA level to 2.89- and 2.02fold that of the control group, respectively (Fig. 5A). Additionally, NF- $\kappa \mathrm{B}$ (1.55-fold of the control group) and TGF- $\beta 1$ (1.88-fold of the control group) mRNA levels were significantly upregulated by HS, whereas $\beta$-CA co-treatment significantly decreased the NF- $\kappa$ B and TGF- $\beta 1$ mRNA levels to 0.96- or 1.17-fold relative to that of the control group, respectively ( $p<0.05$; Fig. 5B and C). As shown in Fig. 5D, BclxL mRNA expression remarkably decreased, by 0.37 -fold, after HS treatment relative to the control group $(p<0.05)$, but that reduced $\mathrm{Bcl}-\mathrm{xL}$ mRNA level was significantly restored 
by co-treatment with EA or $\beta$-CA (0.72- and 0.93-fold, respectively; $p<0.05)$ relative to the control group $(1.0$-fold). Moreover, Bax and caspase $3 \mathrm{mRNA}$ levels in the HS group increased significantly by 3.83 - and 3.26 -fold, respectively, over that of the control group $(p<0.05)$, while co-treatment with EA or $\beta$-CA significantly decreased the Bax mRNA level to 1.78 - and 1.49-fold, respectively, and the caspase 3 mRNA level to 2.45 - and 0.98 -fold, respectively, relative to the control group (1.0-fold) $(p<0.05$; Fig. $5 \mathrm{E}$ and F).

\section{Discussion}

Increasing male infertility and low birth rate are becoming widespread problems. Although a variety of methods and agents have been utilized to cure male infertility, effective and economical natural materials for maintenance of normal spermatogenesis in the presence of various testicular toxicants are lacking. Sex-determining region Y (Sry) and SOX9 have critical roles in male sexual development in mammals, including mice and humans [24]. Moreover, mammalian spermatogenesis progresses from the base to the lumen of the seminiferous tubule. However, because the developmental duration of human testicular epithelia (6 stages) is longer than that of mouse epithelia (12 stages), studies of mouse spermatogenesis have provided more details than those from human studies [25]. On that basis, we conducted a sensitive, exacting experiment using mouse testes to search for a novel natural protective agent against male infertility.

Ellagic acid is a natural polyphenol that originates from plants and has antioxidative and anti-apoptotic properties that function through the chelation of metal ions, which prevents iron- and copper-catalyzed formation of ROS in testes that have been treated with cisplatin [26]. Moreover, $\beta$-CA has been shown to be a potent free radical and singlet oxygen scavenger [18]. Therefore, $\beta-\mathrm{CA}$ and EA could be considered good natural candidates for the protection of testes from various environmental toxicants.

Heat stress causes severe spermatogenic disorders such as desquamation and degeneration of spermatogenic cells and size reduction in seminiferous tubules [27]. Cryptorchidism is a clinical testicular HS-related condition (a physiological HS) that occurs because the testes do not descend into the scrotum and instead remain in the abdominal cavity innately, resulting in abnormal testicular function and spermatogenic disorders [28]. In addition, exogenous scrotal HS (a supraphysiologic HS) that occurs when the testicle is exposed to an external environmental temperature much higher than body temperature can obstruct spermatogenesis, causing spermatogenic cell death and subfertility [8]. Both types of testicular heating induce histological changes in the testes such as apoptotic germ cell death, suggesting that apoptosis can be a major mechanism of impaired spermatogenesis caused by HS [29]. In the present study, mice exposed to transient scrotal hyperthermia had lower relative testes weights than control mice. Furthermore, the HS group showed atrophic changes in the seminiferous tubules including degeneration, reduction and disorganization of germ cells, appearance of multinucleated giant cells, small to large vacuoles and irregular spaces in the epithelium, and degenerative LCs in interstitial tissues. Moreover, a rise in testicle temperature can affect male fertility by decreasing testes weight. These results correspond to those acquired in cryptorchid and acute heat-treated rats [30]. However, compared to the control group co-treatment of HS and $\beta$-CA recovered the HSreduced relative testicular weight significantly. We also demonstrated that HS provoked severe spermatogenic disorders, but these testicular injuries were greatly improved by $\beta$ CA co-treatment.

The testes are very sensitive to lipid peroxidation due to the abundance of polyunsaturated fatty acid. Moreover, spermatogenesis, high rates of cell division, and steroidogenesis of LCs in the testes are susceptible to ROS that cause damages to membranes, proteins, RNA, and DNA [7]. Thus, normal testicular function must be protected against oxidative stress. The testes express various antioxidants such as SOD, GPx, and catalase, which have the ability to act as ROS scavengers of superoxide ion and hydrogen peroxide [31]. In rat testes exposed to HS, increased hydrogen peroxide and lipid peroxidation were accompanied by decreased activities of SOD and catalase [32]. PHGPx is expressed at higher levels in the testes than in other organs, and low PHGPx has been observed in cryptorchidic mice [33]. In the current study, remarkable PHGPx immunostaining was detected in round and elongated spermatids and immature spermatozoa. Such distributional results are correlated with the localization of PHGPx in the testes [33]. However, in the HS group, PHGPx immunoreactivity was mainly detected in degenerative germ cells. The present results support a previous observation that degenerative germ cells are formed by aggregation with round spermatids [34]. Pino et al. [35] showed that compared to pachytene spermatocytes, round spermatids have more antioxidant ability at $40^{\circ} \mathrm{C}$, suggesting that pachytene spermatocytes are more vulnerable to ROS related to high temperatures, but that temperature-resistant mitochondrial uncoupling and high antioxidant ability protects round spermatids from high temperatures. Conversely, the testicular injuries induced by HS were less severe in the HS+EA group than in the HS group, and PHGPx and MnSOD expression patterns in the HS $+\beta-C A$ group were similar to those of the control group. Testes exposed to HS also showed increased MDA levels and reduced PHGPx and MnSOD mRNA expressions and lowered SOD activity. However, EA or $\beta$-CA co-treatment significantly improved these abnormal levels, to those similar to control levels. Indeed, co-treatment with $\beta$-CA further reduced MDA levels and increased the PHGPx mRNA and SOD activity levels in comparison with those of the HS+EA group. These results suggest that co-treatment with EA or $\beta-\mathrm{CA}$ could protect the testes from HS-induced oxidative stress via functional activation of the representative testicular antioxidative enzymes, PHGPx and SOD, and that $\beta$-CA might be a 
potent antioxidant for improvement of spermatogenic disorders induced by HS.

Mild scrotal HS has been shown to be associated with transient blood-testis barrier disruption and the induction of TGF- $\beta$ expression [36]. Scrotal hyperthermia causes hypoxia by reducing the blood flow in the testes [3]. HIF-1 $\alpha$, which is a hypoxia-inducible protein, responds to changes in oxygen level [37], and HS was reported to produce an increase in HIF- $1 \alpha$ mRNA and apoptosis [4]. NF- $\kappa B$ is a redox-sensitive transcription factor that participates in the inflammation process by binding the HIF- $1 \alpha$ promoter under hypoxic states, resulting in apoptosis [38]. Heat stress eventually leads to intrinsic or extrinsic apoptosis of germ cells, and Bcl-2 family proteins such as anti-apoptotic proteins (Bcl-2, Bcl-xL, etc.), pro-apoptotic proteins (Bax and Bak), and apoptogenic proteins (Bad, NF- $\kappa$ B, TGF- $\beta 1$, etc.) regulate apoptosis inside the cell [10]. In previous studies, EA inhibited the lipid peroxidation and apoptotic cell death of testis caused by cyclophosphamide. In addition, the expression of apoptosis-related genes was reduced when testes were pretreated with $\beta$-CA [39]. In the current study, Bcl-xL mRNA levels decreased in response to HS, but recovered significantly in response to cotreatment of HS with EA or $\beta$-CA. Moreover, mRNA levels of HIF- $1 \alpha$, NF- $\kappa$ B, TGF- $\beta 1$, Bax, and caspase 3 were significantly higher after HS stimulation, but co-treatment with EA or $\beta$-CA significantly reduced the elevated mRNA expressions of hypoxia and apoptosis-related proteins, suggesting that the protective effects of $\beta$-CA and EA against HSinduced testicular toxicity might be involved in anti-apoptotic functions through modulation of hypoxia and NF- $\kappa \mathrm{B}$ expression, as well as via the $\mathrm{Bcl}-2$ and caspase 3 pathways.

In testes, testosterone is mainly produced in LCs and has an important role in spermatogenesis. However, testosterone production is seriously affected by HS in the testes [12]. The synthesis of testosterone in LCs relies on the expression of extremely regulated genes such as $3 \beta-\mathrm{HSD}$ and $17 \beta-\mathrm{HSD}$ [40]. In the current study, scrotal HS induced an abnormal production of PHGPx in LCs. Furthermore, serological testosterone levels and testicular $3 \beta$-HSD mRNA expression were greatly reduced in the HS group. However, $\beta$-CA or EA co-treatment significantly restored these decreases to levels almost equal to those of the control group; although $\beta$-CA showed higher androgen biosynthesis ability than EA in testes exposed to HS. These results suggest that $\beta-\mathrm{CA}$ and EA improve abnormal functioning of LCs associated with scrotal hyperthermia by recovering $3 \beta$-HSD and testosterone synthesis in LCs, subsequently leads to normal spermatogenesis.

Although high doses of $\beta$-CA (up to $180 \mathrm{mg}$ /day) have been used without toxic side effects, humans are recommended to consume 3-6 mg $\beta-\mathrm{CA} /$ day to reduce their risk of chronic diseases [15]. According to this study, treatment with $0.3-0.4 \mathrm{mg} \quad \beta-\mathrm{CA} /$ day repaired the hyperthermic damage caused in mouse testes. However, further studies to confirm this via different routes of administration should be conducted.

In conclusion, spermatogenic disorders induced by scrotal
HS were greatly reduced by $\beta$-CA treatment, but only slightly reduced by EA treatment. These findings indicate that $\beta-\mathrm{CA}$ can potently protect against testicular injuries induced by scrotal hyperthermia through its modulation of oxidative stress, apoptosis, and androgen biosynthesis, and may offer a useful preventive and therapeutic choice for the treatment of male infertility caused by environmental toxicants.

\section{Acknowledgments}

This research was supported by the Basic Science Research Program (2016R1D1A1B01015625) through the National Research Foundation of Korea (NRF) funded by the Ministry of Education, Science and Technology and the Research Year of Chungbuk National University in 2018.

\section{References}

1. Chowdhury AK, Steinberger E. Early changes in the germinal epithelium of rat testes following exposure to heat. J Reprod Fertil 1970;22:205-212.

2. Eisenberg ML, Lipshultz LI. Varicocele-induced infertility: newer insights into its pathophysiology. Indian J Urol 2011; 27:58-64.

3. Garolla A, Torino M, Sartini B, Cosci I, Patassini C, Carraro U, Foresta C. Seminal and molecular evidence that sauna exposure affects human spermatogenesis. Hum Reprod 2013; 28:877-885.

4. Ivell R. Lifestyle impact and the biology of the human scrotum. Reprod Biol Endocrinol 2007;5:15.

5. Sheynkin Y, Welliver R, Winer A, Hajimirzaee F, Ahn H, Lee K. Protection from scrotal hyperthermia in laptop computer users. Fertil Steril 2011;95:647-651.

6. Shiraishi K, Takihara H, Matsuyama H. Elevated scrotal temperature, but not varicocele grade, reflects testicular oxidative stress-mediated apoptosis. World J Urol 2010;28: 359-364.

7. Aitken RJ, Roman SD. Antioxidant systems and oxidative stress in the testes. Adv Exp Med Biol 2008;636:154-171.

8. Paul C, Teng S, Saunders PT. A single, mild, transient scrotal heat stress causes hypoxia and oxidative stress in mouse testes, which induces germ cell death. Biol Reprod 2009;80: 913-919.

9. Reyes JG, Farias JG, Henríquez-Olavarrieta S, Madrid E, Parraga M, Zepeda AB, Moreno RD. The hypoxic testicle: physiology and pathophysiology. Oxid Med Cell Longev 2012; 2012:929285.

10. Durairajanayagam D, Agarwal A, Ong C. Causes, effects and molecular mechanisms of testicular heat stress. Reprod Biomed Online 2015;30:14-27.

11. Troiano L, Fustini MF, Lovato E, Frasoldati A, Malorni W, Capri M, Grassilli E, Marrama P, Franceschi C. Apoptosis and spermatogenesis: evidence from an in vivo model of testosterone withdrawal in the adult rat. Biochem Biophys Res Commun 1994;202:1315-1321.

12. Aktas C, Kanter M. A morphological study on Leydig cells of scrotal hyperthermia applied rats in short-term. J Mol Histol 2009;40:31-39.

13. Tapanainen JS, Tilly JL, Vihko KK, Hsueh AJ. Hormonal 
control of apoptotic cell death in the testis: gonadotropins and androgens as testicular cell survival factors. Mol Endocrinol 1993;7:643-650.

14. Krinsky NI, Johnson EJ. Carotenoid actions and their relation to health and disease. Mol Aspects Med 2005;26:459-516.

15. Monsen ER. Dietary reference intakes for the antioxidant nutrients: vitamin $\mathrm{C}$, vitamin $\mathrm{E}$, selenium, and carotenoids. J Am Diet Assoc 2000;100:637-640.

16. Suarez EC, Schramm-Sapyta NL. Race differences in the relation of vitamins $\mathrm{A}, \mathrm{C}, \mathrm{E}$, and $\beta$-carotene to metabolic and inflammatory biomarkers. Nutr Res 2014;34:1-10.

17. Bai SK, Lee SJ, Na HJ, Ha KS, Han JA, Lee H, Kwon YG, Chung CK, Kim YM. Beta-carotene inhibits inflammatory gene expression in lipopolysaccharide-stimulated macrophages by suppressing redox-based NF-kappaB activation. Exp Mol Med 2005;37:323-334.

18. El-Missiry MA, Shalaby F. Role of beta-carotene in ameliorating the cadmium-induced oxidative stress in rat brain and testis. J Biochem Mol Toxicol 2000;14:238-243.

19. Usta C, Ozdemir S, Schiariti M, Puddu PE. The pharmacological use of ellagic acid-rich pomegranate fruit. Int $\mathrm{J}$ Food Sci Nutr 2013;64:907-913.

20. Jung KY, Lin C, Yon JM, Jung AY, Park SG, Chu SB, Gwon LW, Nam SY. Postnatal expression profiles of phospholipid hydroperoxide glutathione peroxidase in spermatogenesis in mice. Turk J Vet Anim Sci 2016;40:40-46.

21. Park SG, Yon JM, Lin C, Gwon LW, Lee JG, Baek IJ, Lee BJ, Yun YW, Nam SY. Capsaicin attenuates spermatogenic cell death induced by scrotal hyperthermia through its antioxidative and anti-apoptotic activities. Andrologia 2017;49: e12656.

22. Livak KJ, Schmittgen TD. Analysis of relative gene expression data using real-time quantitative PCR and the 2(-Delta Delta C(T)) Method. Methods 2001;25:402-408.

23. Lin C, Yon JM, Jung AY, Lee JG, Jung KY, Kang JK, Lee BJ, Yun YW, Nam SY. Resveratrol prevents nicotine-induced teratogenesis in cultured mouse embryos. Reprod Toxicol 2012;34:340-346

24. Warr N, Greenfield A. The molecular and cellular basis of gonadal sex reversal in mice and humans. Wiley Interdiscip Rev Dev Biol 2012;1:559-577.

25. Muciaccia B, Boitani C, Berloco BP, Nudo F, Spadetta G, Stefanini M, de Rooij DG, Vicini E. Novel stage classification of human spermatogenesis based on acrosome development. Biol Reprod 2013;89:60.

26. Türk G, Ateşşahin A, Sönmez M, Ceribaşi AO, Yüce A. Improvement of cisplatin-induced injuries to sperm quality, the oxidant-antioxidant system, and the histologic structure of the rat testis by ellagic acid. Fertil Steril 2008;89 Suppl: 1474-1481.

27. Setchell BP. The Parkes Lecture. Heat and the testis. J
Reprod Fertil 1998;114:179-194.

28. Blackshaw AW, Massey PF. The effect of cryptorchidism on the quantitative histology, histochemistry and hydrolytic enzyme activity of the rat testis. Aust J Biol Sci 1978;31:53-64.

29. Wang C, Cui YG, Wang XH, Jia Y, Sinha Hikim A, Lue YH, Tong JS, Qian LX, Sha JH, Zhou ZM, Hull L, Leung A, Swerdloff RS. transient scrotal hyperthermia and levonorgestrel enhance testosterone-induced spermatogenesis suppression in men through increased germ cell apoptosis. J Clin Endocrinol Metab 2007;92:3292-3304.

30. Ren L, Medan MS, Ozu M, Li C, Watanabe G, Taya K. Effects of experimental cryptorchidism on sperm motility and testicular endocrinology in adult male rats. J Reprod Dev 2006;52:219-228.

31. Gu W, Hecht NB. Developmental expression of glutathione peroxidase, catalase, and manganese superoxide dismutase mRNAs during spermatogenesis in the mouse. J Androl 1996; 17:256-262.

32. Ikeda M, Kodama H, Fukuda J, Shimizu Y, Murata M, Kumagai J, Tanaka T. Role of radical oxygen species in rat testicular germ cell apoptosis induced by heat stress. Biol Reprod 1999;61:393-399.

33. Jung KY, Yon JM, Lin C, Jung AY, Lee JG, Baek IJ, Lee BJ, Yun YW, Nam SY. Phospholipid hydroperoxide glutathione peroxidase is involved in the maintenance of male fertility under cryptorchidism in mice. Reprod Toxicol 2015;57:73-80.

34. Anton E. Arrested apoptosis without nuclear fragmentation produced by efferent duct ligation in round spermatids and multinucleated giant cells of rat testis. Reproduction 2003; $125: 879-887$.

35. Pino JA, Osses N, Oyarzún D, Farías JG, Moreno RD, Reyes JG. Differential effects of temperature on reactive oxygen/ nitrogen species production in rat pachytene spermatocytes and round spermatids. Reproduction 2013;145:203-212.

36. Cai H, Ren Y, Li XX, Yang JL, Zhang CP, Chen M, Fan $\mathrm{CH}, \mathrm{Hu} \mathrm{XQ}, \mathrm{Hu} \mathrm{ZY}$, Gao F, Liu YX. Scrotal heat stress causes a transient alteration in tight junctions and induction of TGF- $\beta$ expression. Int J Androl 2011;34:352-362.

37. Choi KS, Bae MK, Jeong JW, Moon HE, Kim KW. Hypoxia-induced angiogenesis during carcinogenesis. J Biochem Mol Biol 2003;36:120-127.

38. Görlach A, Bonello S. The cross-talk between NF-kappaB and HIF-1: further evidence for a significant liaison. Biochem J 2008;412:e17-e19.

39. Türk G, Ceribaşi AO, Sakin F, Sönmez M, Ateşşahin A. Antiperoxidative and anti-apoptotic effects of lycopene and ellagic acid on cyclophosphamide-induced testicular lipid peroxidation and apoptosis. Reprod Fertil Dev 2010;22:587-596.

40. Rizk SM, Zaki HF, Mina MA. Propolis attenuates doxorubicininduced testicular toxicity in rats. Food Chem Toxicol 2014;67:176-186. 